\title{
Development of an Improved Feature based Algorithm for Image Matching
}

\author{
Kanwalvir Singh Dhindsa \\ Punjab Technical University \\ BBSBEC Fatehgarh Sahib
}

\author{
Geetanjali Babbar \\ Punjab Technical University \\ BBSBEC Fatehgarh Sahib
}

\begin{abstract}
Image matching plays an important role in many fields such as pattern searching \& recognizing[6], image analysis, robotics \& computer vision. It is a method to find a certain image in the image database which matches or can be said similar to the given template picture. The template image can be thought of as a subset of the matching image. This paper aims at the improved matching algorithm which is based on the image feature point[5]. By searching correct feature point and setting bidirectional threshold value, the matching process can be quickly \& precisely implemented with optimistic results. Visual $\mathrm{C}++$ to be used for design and implementation. In future, the feature based algorithm can be modified to choose feature selection threshold adaptively depending on the image's content.
\end{abstract}

Key Words: correspondence, image features, convergence, versatility.

\section{INTRODUCTION}

The image matching problem, also known as the correspondence problem[7] is one of the most challenging research task in the computer vision. The basic principle of matching is searching the whole pixel set space for the right area which is identical to the given template image[9]. The applicability of complete search in the image space becomes a costly affair because of large quantity of image pixels in the image space and realistically this cost becomes unbearable. Further for image of $800 \times 600$ pixels, we may spend as long as 4-seconds time in searching the right area by traditional method[2]. In order to lessen the searching time, usually we have to improve our algorithms in wide aspects like reducing the searching space and the time which can finally achieve the results of fast and safety matching.

This paper put forward an improved feature point based matching algorithm. Through the analysis and comparison, the performance and the efficiency is improved. The exact position of objects, units and features related to image must be known [4].If we search such feature point in the whole picture scope, the feature point selected may be too dense as there may be too many feature points in a small area[3], one extremely phenomenon is that the feature points are in one single line in the edge of an object. So we should dispart the picture to sub areas, for example, the NxM pixels image may be partitioned to sub areas each composed of (NxM)/ (nxm) pixels, that means we now get nxm sub areas. Then we can search the feature point in each sub area scope, and in each we just need one feature point, in the end we may get nxm feature points, and these feature points will be distributed averagely, and hence improves the result.

We can now set a threshold to decide if a point is a feature point, if a pixel surpassed this threshold, the pixel can be thought of as a feature point.

\section{BASIC CONCEPTS}

\subsection{Image nature}

Another useful classification is between single-modality and multi-modality matching algorithms. Single modality matching algorithms are those, intended to match images of the same modality (i.e. acquired using the same kind of imaging device); while multi-modality matching algorithms are those intended to match images acquire using different imaging devices.

There are several examples of multi-modality matching algorithms in the medical imaging field. Examples include registration of brain CT/MRI images or whole body PET/CT images against no-contrasting enhanced CT images for segmentation of specific parts of the anatomy and matching of ultrasound and CT images for prostate localization in radiotherapy.

\subsection{Image Matching Algorithms}

The distinction between different matching primitives is probably the most prominent difference between the various matching algorithms. The primitives fall into two broad categories: either windows composed of grey values or features extracted in each image a priori are used in the actual matching step. The resulting algorithms are usually called:

1. Area based matching (ABM), and

2. Feature based matching (FBM), respectively.

Area based matching sometimes called signal based matching is considered more traditional. The cross correlation \& least square matching are well known methods for area based matching. These methods usually require very good initial values for the unknown parameters.

Feature based matching determines the correspondence b/w image features and it does not require very precise initial estimates. In both cases, there is a choice b/w local \& global support for the primitives. Local refers to an area seldom larger than about $15 * 15$ or $21 * 21$ pixel in image space, global means a larger area and can comprise the whole image. Local matching is very precise, but ambiguous \& the various local features may not be consistent with each other. Global matching is more robust but not necessarily accurate in local areas.

In general, algorithms now frequently use constraints on the primitives in order to find an optimal solution. The most common ones are:

1. Epipolar constraint: Two homologous points must respectively be located on the two epipolar lines of the respective object point. This constraint depends on the geometry of the aerial survey and is independent of the scene's content.

2. Uniqueness constraint: The primitive of an image cannot have more than one homologous primitive in the other image. This rule often has exceptions, such as occlusions, transparent 
surfaces or different points that lie on a line, which is being seen as a point.

3. Surface continuity constraint: depending on the surface we can assume e.g. planar surface.

4. Order constraint: if $\mathrm{p} 1$ is on the right of $\mathrm{q} 1$ in image1, then $\mathrm{p} 2$ is also on the right of q2 in the second image. An inversion of points occurs only for objects that present a high perspective deformation on the image.

5. Photometry constraint: based on Lambert's hypothesis, for an object point the reflected light intensity should be the same in all directions. However, many reasons may cause radiometric variations between two overlapping images.

\section{FEATURE BASED MATCHING}

The resemblance of two images is defined as the overall similarity between two families of image features[1]. The feature based matching is based on the following points.

1) To select distinct points in the images separately.

2) The points are selected using a so-called interest operator which has been found to be optimal for feature point detection [8][10].

3) Building up a preliminary list of candidate pairs of corresponding points assuming a similarity measure.

4) Finding the final list of point pairs consistent with an object model.

\subsection{Selecting Distinct Points with an Interest operator}

In Feature based matching instead of matching all pixels in an image, only selected points with certain features are to be matched. The selection principle should fulfill the following requirements:

Distinctness: The points should be distinct, i.e. be different from neighbouring points e.g. points on edges should not be selected if the epipolar geometry constraint is not used; also points in flat areas should not be selected. MORAVEC's and HANNAH's operators follow this aim: MORAVEC's operator searches for points with the largest minimum variance of gray level differences in 4 directions, while HANNAH's operator searches for points where the autocorrelation function of the gray level function is steep in all directions.

Invariance: The selection as well as the selected position should be invariant with respect to the expected geometric and radiometric distortions. This besides the distinctness probably is the most important requirement. The degree of invariance directly influences the precision and the reliability of the matching.

Stability: The selected points should be expected to appear in other images. Thus the selection should be robust with respect to noise. In image sequence analysis the selected points should appear in long sequences of consecutive frames.

Interpretability: The selection principle should be interpretable in some sense, e.g. looking for edges, corners or other simple but labeled features. This requirement is not essential from an engineering point of view, but may be essential if the interest operator is used for image analysis.
Seldomness: Whereas distinctness guarantees local separability of points, seldomness aims at global separability. This is essential in images with partially repetitive patterns.

\subsection{The improved feature based point selection algorithm}

Stepl: If there is any sub area that has not been searched, if true, set the current point to the first point in the sub area which located in the top left, and set D as 0 , if false, end the searching process.

For each point $T(i, j)$ in template image $(T)$, we compute the difference with each neighbour pixel as $\mathrm{D}$ :

$$
D=\sum_{-1 \leq a, b \leq 1} \sum(T(i, j)-T(i+a)(j+b))^{2}
$$

Step2: If the current point is the last one in this sub area, if not: go to step3, else put the point information which has the largest $\mathrm{D}$ value to the feature point array, and back to step 1;

Step3: Compute the D value of the current point, set to SUM, compare SUM to D if $S U M>D$, put the $S U M$ value to D, record the point, move the current point to the next point, go to step3.

Step4: If every sub area has found their feature point, we can get a full feature point array, which now records the information of the feature points, and we can start our matching computing right now. For optimization of feature point selection we have used a feature threshold parameter, which is useful in enhancing the matching speed by carefully selecting/rejecting the feature point to be matched.

\subsection{The Analysis of improved feature based match algorithm}

3.3.1 The efficiency of match algorithm

The image matching has two conditions, one is: the sub image has been resized or rotated; the other is: the size is different but the image proportion is similar. The first condition may have wider usage, but the second condition is the basis of image matching, so it also has theoretical significance. It firstly proposes a template image that is smaller than the matching image, even it is just obtained from the matching image, we can treat it as the sub image of the matching image and we call this algorithm as same-proportion image matching algorithm. In majority same-proportion image matching algorithm, always compare the template image to the sub image of the matching image which have the same size, and by analyzing the difference we can get the result of matching or mismatching. The common method is by setting a threshold, and if the difference is in the scope of the threshold, we think the matching is successful. Through this strategy, if there exists a matching area, the search will finally be successful.

To a $800 \times 600$ pixels matching image, if the template image is $40 \times 40$ pixels, by the traditional template matching algorithm, the comparing round is $(800-40+1) \times(600-40+1)$, and in each round, we should compute $40 \times 40$ times, so the computation is huge. The image matching strategy based on feature point can largely reduce the computation. For a template image of $40 \times 40$, we can divide it into $4 \times 4$ sub area, by the selection of 16 feature points, we can shorten the work to $1 \%$. 


\subsubsection{The selection of match threshold}

At first we should set a threshold, it can then be the standard of the feature point matching. This threshold can be set according to the matching application. For example, to those images which have variety color changing, we should set a larger threshold, and to those images which are composed of similar color, and identical texture, we should set a lower threshold, and otherwise we may find several wrong but similar image results.

\section{THE RESULTS}

Matching has been performed using feature based matching and area based matching and their image matching time (per second) is calculated to evaluate the performance and their behavior, as shown in the figures taken from the database. The figure 1 is the simple template image and figure 2 is the noisy image.

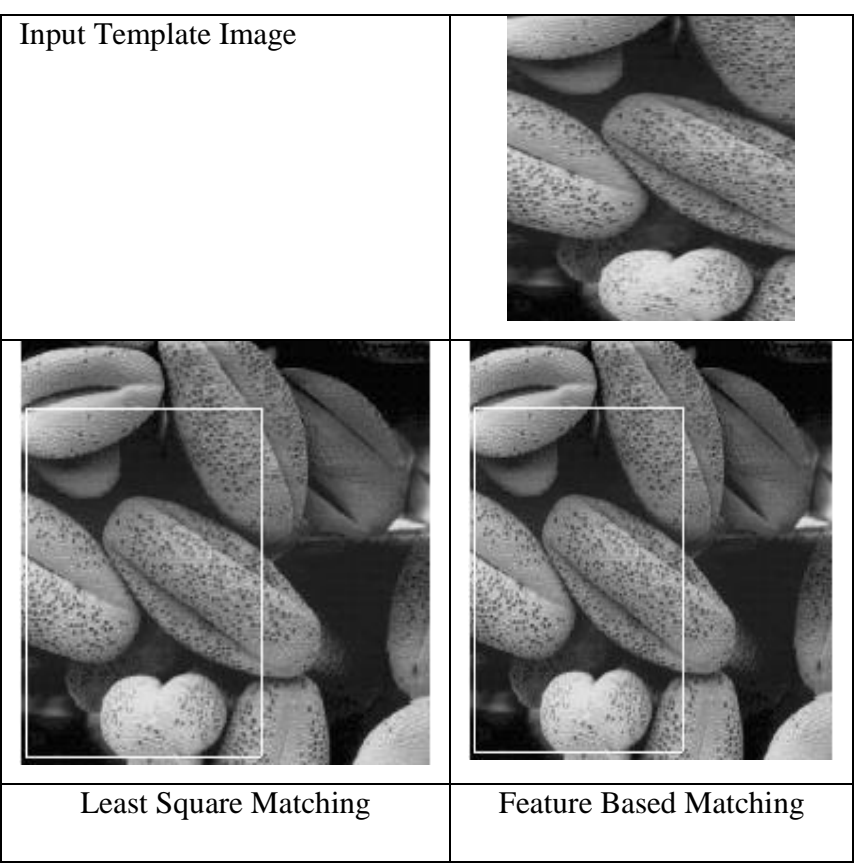

Figure 1: Output after matching

Table1. Time taken (in seconds) by FBM and ABM matching techniques. Feature Based Matching matching has been performed at different threshold levels.

\begin{tabular}{|c|c|c|c|c|c|c|}
\hline \multicolumn{5}{|c|}{ Feature Based Matching } & $\begin{array}{c}\text { Area Based } \\
\text { Matching }\end{array}$ \\
\cline { 1 - 4 } $\begin{array}{c}\text { S. } \\
\text { No. }\end{array}$ & NOF & RT & WT & FBM Time & $\begin{array}{c}\text { Feature } \\
\text { Threshold }\end{array}$ & $\begin{array}{c}\text { LSM Time } \\
\text { seconds }\end{array}$ \\
\hline 1 & \multirow{2}{*}{15} & 0.9 & 0.1 & 0.156000 & \multirow{2}{*}{10000} & \multirow{2}{*}{0.437000} \\
\cline { 4 - 6 } & 0.8 & 0.2 & 0.156000 & & \\
\cline { 4 - 6 } 4 & \multirow{2}{*}{10} & 0.9 & 0.1 & 0.110000 & \multirow{2}{*}{15000} & \\
\cline { 3 - 5 } 5 & 0.8 & 0.2 & 0.110000 & & \\
\hline
\end{tabular}

NOF: No. of Features

RT: Right Threshold (True Threshold)

WT: Wrong Threshold
Table2. The experimental data can be concluded with following parameters:

\begin{tabular}{|c|c|c|}
\hline Parameters & FBM & ABM \\
\hline Algorithm & $\begin{array}{l}\text { Same proportion image } \\
\text { matching algorithm using } \\
\text { bi-directional threshold } \\
\text { image matching } \\
\text { technique is used. }\end{array}$ & $\begin{array}{l}\text { Both Cross } \\
\text { correlation \& Least } \\
\text { square matching } \\
\text { can be used but in } \\
\text { example the results } \\
\text { are derived with } \\
\text { LSM. }\end{array}$ \\
\hline $\begin{array}{ll}\text { No. of } \\
\text { Features }\end{array}$ & $\begin{array}{l}\text { The No. of features taken } \\
\text { are } 15 \text { or } 10 .\end{array}$ & $\begin{array}{l}\text { The No. of features } \\
\text { taken are } 15 \text { or } 10 .\end{array}$ \\
\hline $\begin{array}{l}\text { Time } \\
\text { taken(in } \\
\text { seconds) }\end{array}$ & $\begin{array}{l}\text { The time taken for the no. } \\
\text { of features } 10 \text { is } 0.156000 \\
\text { and for the no. of features } \\
15 \text { is } 0.110000\end{array}$ & $\begin{array}{l}\text { The time taken is } \\
0.437000 \text { which is } \\
\text { more than the } \\
\text { feature based } \\
\text { matching. }\end{array}$ \\
\hline
\end{tabular}

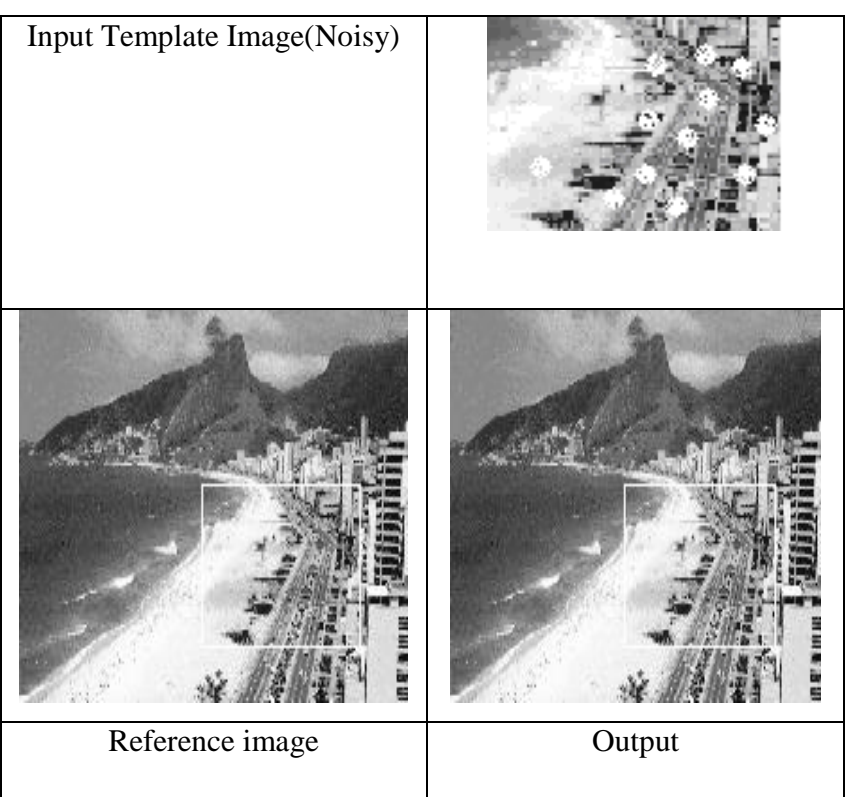

Figure 2: Output after matching (noisy image is taken). After Area Based Matching and Feature Based Matching no patch has been found. As a result of this no sub image area is marked.

\section{COMPARITIVE FEATURES OF AREA BASED \& FEATURE BASED MATCHING}

\begin{tabular}{|c|c|c|}
\hline Parameters & $\begin{array}{c}\text { Area based } \\
\text { Matching }\end{array}$ & $\begin{array}{c}\text { Feature Based } \\
\text { Matching }\end{array}$ \\
\hline
\end{tabular}




\begin{tabular}{|c|c|c|}
\hline $\begin{array}{l}\text { 1. Matching } \\
\text { Strategy }\end{array}$ & $\begin{array}{l}\text { Small window of } \\
\text { pixels in a } \\
\text { reference image } \\
\text { (template) is } \\
\text { compared with } \\
\text { equally sized } \\
\text { windows of pixels } \\
\text { in other (target) } \\
\text { images. }\end{array}$ & $\begin{array}{l}\text { In FBM, instead } \\
\text { of matching all } \\
\text { pixels in an } \\
\text { image, only } \\
\text { selected points } \\
\text { with certain } \\
\text { features are to be } \\
\text { matched. }\end{array}$ \\
\hline $\begin{array}{l}\text { 2. Convergence, } \\
\text { Speed \& } \\
\text { Versatility }\end{array}$ & $\begin{array}{l}\text { ABM is being } \\
\text { observed with less } \\
\text { Convergence, } \\
\text { speed and } \\
\text { versatility as } \\
\text { compared to } \\
\text { FBM. }\end{array}$ & $\begin{array}{l}\text { FBM is superior } \\
\text { with respect to } \\
\text { speed, versatility, } \\
\text { and range of } \\
\text { convergence. }\end{array}$ \\
\hline $\begin{array}{l}\text { 3. Initial } \\
\text { Estimates }\end{array}$ & $\begin{array}{l}\text { ABM usually } \\
\text { requires very good } \\
\text { initial values for } \\
\text { the unknown } \\
\text { parameters. }\end{array}$ & $\begin{array}{l}\text { FBM does not } \\
\text { require very } \\
\text { precise initial } \\
\text { estimates. }\end{array}$ \\
\hline 4. Sensitivity & $\begin{array}{l}\text { More sensitive } \\
\text { with respect to the } \\
\text { quality } \\
\text { approximate } \\
\text { values. }\end{array}$ & $\begin{array}{l}\text { Less sensitive } \\
\text { with respect to the } \\
\text { quality of } \\
\text { approximate } \\
\text { values. }\end{array}$ \\
\hline 5. Occlusions & $\begin{array}{l}\mathrm{ABM} \text { is sensitive } \\
\text { with respect to } \\
\text { occlusions. }\end{array}$ & $\begin{array}{l}\text { Less sensitive } \\
\text { with respect to } \\
\text { occlusions. }\end{array}$ \\
\hline $\begin{array}{l}\text { 6. Illumination } \\
\text { changes }\end{array}$ & $\begin{array}{l}\text { Don't work well } \\
\text { when viewpoints } \\
\text { are very different } \\
\text { due to change in } \\
\text { illumination } \\
\text { direction. }\end{array}$ & $\begin{array}{l}\text { Relatively } \\
\text { insensitive } \\
\text { illumination } \\
\text { changes. }\end{array}$ \\
\hline 7. Speed & Slow & Fast \\
\hline 8. Disparity & $\begin{array}{l}\text { Provide a dense } \\
\text { disparity map }\end{array}$ & $\begin{array}{l}\text { Provide sparse } \\
\text { disparity map }\end{array}$ \\
\hline
\end{tabular}

The results of experimental data and comparative study states that feature based matching is superior to LSM because it takes less time for the matching process.

\section{CONCLUSIONS}

In this paper we have taken the results with feature Based matching (same proportion image matching algorithm using bi-directional threshold) and area based matching (cross correlation and least square matching).

From the experiments that are given in result section, it has been observed that normally feature based matching algorithm is faster in comparison to the area based matching technique. FBM time complexity depends on number of features selected as well as the right and wrong threshold. If the numbers of features are high then sometimes it takes more computational time in comparison to $\mathrm{ABM}$ techniques.

\section{FUTURE SCOPE}

In future more work can be carried out to have adaptive feature selection method. The number of features extracted from an image depends largely on the contents of an image. If there are high variations then features computed are high. This reduces time efficiency to match. In future the feature based algorithm can be modified to choose feature selection threshold adaptively depending upon the image content.

\section{REFERENCES}

[1] R. C. Joshi, and Shashikala Tapaswi, "Image Similarity: A Genetic Algorithm Based Approach", Proceedings of world academy of science, engineering and technology volume 21 MAY 2007 ISSN 1307-6884

[2] Hu Minghao, Ren Mingwu, Yang Jingyu, “ A rapid and useful algorithm of image matching based on Feature Point", computer engineering 2004.5 Vol.30

[3] Li Junshan, Shen Xubang,"the research of image matching technology", Micro-electric and computer 2002-2

[4] Abdul Ghafoor, Rao Naveed Iqbal, and Shoab Khan, "Robust image matching algorithm", EC-VIP-MC $2003,4^{\text {th }}$ EURASIP Conference focused on Video/Image Processing and Multimedia Communications, 2-5 July 2003, Zagreb, Croatia.

[5] Dare P M, Dow man, "A Comparison of feature extraction algorithms for automated feature based multisensor image registration", IJRSS'97, 1997

[6] Gu Hui, Chen Guangyi and Cao Wenming, "Image Matching Algorithm Based on Feature Point with Bidrectional Threshold", College of Information Engineering, Zhejiang University of Technology, Hangzhou 310031, Zhenjiang, China, 2005 IEEE.

[7] Hassan Hajjdiab and Robert Laganiere, "Complexity Analysis of Feature- Based Image Matching", World Academy of Science, Engineering and Technology 51, 2009.

[8] Ida JAZAYERI, Simon CRONK and Clive FRASER, "A Feature Based Matching Approach to Automated Object Reconstruction in Multi-Image Close-Range Photogrammetry", FIG Congress, Facing the Challenges - Building the Capacity, Sydney, Australia, 11-16 April 2010.

[9] Weili JIAO, Yaling FANG, Guojin, "An Integrated Feature Based Method for sub-pixel Image matching", the International Archives of the Photogrammetry, Remote Sensing and Spatial Information Sciences. Vol. XXXVII. Part B1. , Beijing 100086, CHINA, 2008.

[10] Jazayeri, I., Fraser, "Interest Operators for Feature-Based Matching in Close-Range Photogrammetry". Photogrammetric Record (in press) C.S. (2009). 